\title{
Lorenzo's Oil
}

National Cancer Institute

\section{Source}

National Cancer Institute. Lorenzo's Oil. NCI Thesaurus. Code C121561.

An orally-administered oil composed of the monounsaturated fatty acids glyceryl trioleate (GTO), the triacylglycerol form of oleic acid derived from olive oil, and glyceryl trierucate (GTE), the triacylglycerol form of erucic acid derived from rapeseed oil, in a 4:1 ratio, that can be used in the treatment of adrenoleukodystrophy (ALD). Upon oral administration of Lorenzo's oil, the monounsaturated fatty acids compete with saturated fatty acids as substrates for enzymes in the elongation of very long-chain fatty acids (ELOVL) family. This results in the elong ation and production of monounsaturated very long-chain fatty acids (VLCFAs) and reduces the elong ation of saturated VLCFAs. This reduces the plasma levels of saturated VLCFAs and prevents their accumulation in the brain, thereby preventing demyelination of cerebral nerves and halting the progression of ALD. 